Forum 2021 · 36:7

https://doi.org/10.1007/s12312-020-00882-0

Online publiziert: 5. Januar 2021

(c) Springer Medizin Verlag GmbH, ein Teil von Springer Nature 2020

Systematische Qualitätsentwicklung und Versorgungsforschung in der Onkologie zählen zu den Themen, die die Deutsche Krebsgesellschaft seit vielen Jahren erfolgreich vorantreibt. Seit 2013 richten wir gemeinsam mit der Arbeitsgemeinschaft Deutscher Tumorzentren (ADT) alle zwei Jahre eine Veranstaltung aus, die sich an interessierte Expert ${ }^{*}$ innen aus diesen Bereichen wendet, den Interdisziplinären Kongress Quality of Cancer Care (QoCC). Ich freue mich sehr, als Präsident der DKG gemeinsam mit der Vorstandsvorsitzenden der ADT, Prof. Monika Klinkhammer-Schalke, den QoCC-Kongress im nächsten Jahr eröffnen zu dürfen. Unter dem Motto "Qualität in der onkologischen Chirurgie" findet die Veranstaltung erstmals digital statt: Die Teilnehmer*innen erwartet nicht nur ein spannendes Vortragsangebot am 18./19. Februar; bereits ab dem 20. Januar können Sie in insgesamt neun Online-Tutorials und neun digitalen Posterbegehungen ihr Wissen erweitern und aktuelle Forschungsergebnisse diskutieren.

Warum haben wir uns dazu entschlossen, dieses Mal die onkologische Chirurgie in den Mittelpunkt zu stellen? Wer die Presse aufmerksam verfolgt, wird mir zustimmen: Über innovative medikamentöse Krebstherapien berichten die Medien häufig, über die Bedeutung der Operation für den Heilungsprozess

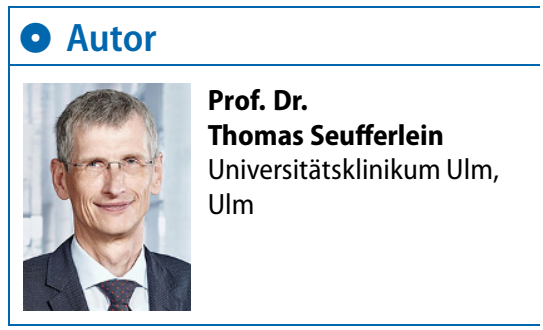

\title{
Thomas Seufferlein
}

Klinik für Innere Medizin I, Universitätsklinikum Ulm, Ulm, Deutschland

\section{Qualität und Interdisziplinarität in der Onkologie}

eher selten. Und das, obwohl $80 \%$ aller Krebspatient*innen im Laufe ihrer Erkrankung operiert werden und die Chirurgie mittlerweile sogar einen festen Platz in der Palliativversorgung hat. Nur ein sehr geringer Teil aller publizierten randomisierten kontrollierten Studien (RCT) vergleichen chirurgische Maßnahmen, Neuerungen bei Operationstechniken müssen nicht zugelassen werden und drängen oft unkontrolliert auf den Markt. Darüber hinaus gelten chirurgische Studien als komplex; oft mangelt es an der Finanzierung und die Zeit, die der chirurgische Arbeitsalltag für Forschung übriglässt, ist knapp bemessen. Beim QoCC-Kongress 2021 wird es unter anderem um den Nutzen der Spezialisierung in der chirurgischen Onkologie gehen, um neue Wege zur Implementierung onkologisch-chirurgischer Forschung, um die Qualitätssicherung anhand von Registern und Studien am Beispiel des Pankreaskarzinoms.

Ohne gute und praktikable Ansätze für eine interdisziplinäre und sektorenübergreifende Krebsversorgung laufen diese Bestrebungen ins Leere. Deshalb betrachten wir die onkologische Chirurgie nicht als isolierte Einzeldisziplin, sondern eingebettet in den Kontext multimodaler onkologischer Maßnahmen. Ich freue mich sehr auf die Zusammenschau aller wichtigen Aspekte in unserem onkologischen Qualitätszyklus - von der Erstellung evidenzbasierter Leitlinien über die Zertifizierung und Dokumentation bis hin $\mathrm{zu}$ klinischen Studien sowie der Versorgungsforschung. Besondere Aufmerksamkeit verdient unter anderem die Frage, wie sich die interdisziplinäre Zusammenarbeit aller an der Krebsversorgung beteiligten Disziplinen an den Übergängen der Stationen dieses
Qualitätszyklus optimal und ohne Reibungsverluste gestalten lässt. In diesem Zusammenhang haben wir verschiedene digitale Klassen ins Programm integriert, in denen die neuesten Entwicklungen in unserem Leitlinienprogramm Onkologie, in den zertifizierten Zentren und den Krebsregistern vorgestellt werden. Auch wenn wir 2021 pandemiebedingt auf eine Live-Veranstaltung verzichten, unser Online-Veranstaltung bietet ein abwechslungsreiches flexibles Format, das Sie sich auf keinen Fall entgehen lassen sollten.

Ihr Thomas Seufferlein Präsident der Deutschen Krebsgesellschaft

\section{Korrespondenzadresse}

Prof. Dr. Thomas Seufferlein

Klinik für Innere Medizin I, Universitätsklinikum UIm

Albert-Einstein-Allee 23, 89081 Ulm,

Deutschland

Thomas.seufferlein@uniklinik-ulm.de

Interessenkonflikt. T. Seufferlein gibt an, dass kein Interessenkonflikt besteht. 